TRANSACTIONS OF THE

AMERICAN MATHEMATICAL SOCIETY

Volume 351, Number 12, Pages 4823-4835

S 0002-9947(99)02188-1

Article electronically published on August 20, 1999

\title{
EXAMPLES OF MÖBIUS-LIKE GROUPS WHICH ARE NOT MÖBIUS GROUPS
}

\author{
NATAŠA KOVAČEVIĆ
}

\begin{abstract}
In this paper we give two basic constructions of groups with the following properties:

(a) $G \hookrightarrow \mathrm{Homeo}_{+}\left(S^{1}\right)$, i.e., the group $G$ is acting by orientation preserving homeomorphisms on $S^{1}$;

(b) every element of $G$ is Möbius-like;

(c) $L(G)=S^{1}$, where $L(G)$ denotes the limit set of $G$;

(d) $G$ is discrete;

(e) $G$ is not a conjugate of a Möbius group.

Both constructions have the same basic idea (inspired by Denjoy): we start with a Möbius group $H$ (of a certain type) and then we change the underlying circle upon which $H$ acts by inserting some closed intervals and then extending the group action over the new circle. We denote this new action by $\bar{H}$. Now we form a new group $G$ which is generated by all of $\bar{H}$ and an additional element $g$ whose existence is enabled by the inserted intervals. This group $G$ has all the properties (a) through (e).
\end{abstract}

\section{INTRODUCTION}

Denote by $\mathcal{M}$ the group of orientation preserving Möbius transformations of the complex plane preserving the unit disc. Every element of $\mathcal{M}$ also preserves the boundary, $S^{1}$, of the unit disc. In other words, we can view $\mathcal{M}$ as a subgroup of the group of all orientation preserving homeomorphisms of $S^{1}$ via

$$
\mathcal{M} \hookrightarrow \text { Homeo }\left._{+}\left(S^{1}\right) f \longmapsto f\right|_{S^{1}},
$$

where $\left.f\right|_{S^{1}}$ denotes the restriction of $f$ to $S^{1}$. Throughout this paper we will refer to Möbius groups as subgroups of $\mathcal{M}$, and we will mostly think of them in terms of their restrictions to $S^{1}$. Fuchsian groups are Möbius groups which are discrete (in the compact-open topology).

Definition. Given $f \in \mathrm{Homeo}_{+}\left(S^{1}\right)$ we say that $f$ is Möbius-like if there exists some homeomorphism $g$ of $S^{1}$ such that $g f g^{-1} \in \mathcal{M} .{ }^{1}$ Accordingly, given a group $G \hookrightarrow \mathrm{Homeo}_{+}\left(S^{1}\right)$, we say that $G$ is Möbius-like if every element of $G$ is a Möbiuslike map.

The main purpose of this paper is to give some interesting examples of group actions on the circle. Namely we exhibit two basic constructions of groups with the following properties:

Received by the editors March 7, 1995 and, in revised form, July 31, 1997.

1991 Mathematics Subject Classification. Primary 57S05.

${ }^{1}$ Equivalently, $f$ is Möbius-like if it has the dynamics of a Möbius transformation.

(C)1999 American Mathematical Society 
(a) $G \hookrightarrow \mathrm{Homeo}_{+}\left(S^{1}\right)$, i.e., the group $G$ is acting by orientation preserving homeomorphisms on $S^{1}$;

(b) every element of $G$ is Möbius-like;

(c) $L(G)=S^{1}$, where $L(G)$ denotes the limit set of $G$;

(d) $G$ is discrete;

(e) $G$ is not a conjugate of a Möbius group.

Both constructions have the same basic idea: we start with a Möbius group $H$ and then we change the underlying circle upon which $H$ acts by inserting some closed intervals and then extending the group action over the new circle. We denote this new action by $\bar{H}$. Now we form a new group $G$ which is generated by all of $\bar{H}$ and an additional element $g$ whose existence is enabled by the inserted intervals. This group $G$ will have the property that its every element is Möbius-like, provided that the initial group $H$ was from a specific class of Möbius groups.

This specific class of Möbius groups is naturally divided into two subclasses corresponding to two distinct ways of carrying out the above outline. That is why we talk about two constructions. Namely, the initial group $H$ has to be one of the following two types:

1. Nondiscrete elementary countable Möbius group without global fixed points;

2. Fuchsian group of the first kind which has at least one approximation point on the circle which is not fixed by any element of $H$.

It should be noted that in both cases the terminal group $G$ has no global fixed points. This is no coincidence since the characterization of groups with at least one global fixed point and all of whose elements are Möbius-like is known : such a group is a conjugate of a Möbius group if and only if its limit set is all of the circle. See $[K]$.

\section{PRELIMINARY DISCUSSIONS}

Throughout this paper we restrict ourselves to orientation preserving homeomorphisms of the circle only.

View $S^{1}$ as being oriented counterclockwise; therefore $(x, y)$ denotes all the points counterclockwise from $x$ to $y$. We define $[x, y),(x, y],[x, y]$ similarly. Given two open intervals $(x, y)$ and $(u, v)$ on $S^{1}$ we write $(x, y) \sim(u, v)$ if either $x<u<y<$ $v<x$ or $u<x<v<y<u$; see Figure 1 .

The universal cover of $S^{1}$ is $\mathbf{R}$ via the covering map $x \mapsto e^{2 \pi i x}, x \in \mathbf{R}$. By abuse of notation we denote all lifts to $\mathbf{R}$ of a point $x \in S^{1}$ by the same symbol $x$.

Given $f \in$ Homeo $_{+}\left(S^{1}\right)$, it can be lifted to a homeomorphism $\tilde{f}: \mathbf{R} \rightarrow \mathbf{R}$ so that $f\left(e^{2 \pi i x}\right)=e^{2 \pi i \tilde{f}(x)}, \forall x \in \mathbf{R}$. Since $f$ is orientation preserving, $\tilde{f}$ is a strictly increasing function. There are many different lifts of $f$, but we will most commonly take $\tilde{f}$ to be the lift of $f$ whose graph is "the closest" to the line $y=x$. Actually, by abuse of notation we will use $f$ for $\tilde{f}$.

Some arguments that follow deal with functions $S^{1} \rightarrow S^{1}$ which are limits of sequences of homeomorphisms of $S^{1}$. It is therefore natural to introduce the following convention about drawing the graphs of such functions (i.e., their lifts to $\mathbf{R}$ ): if a function $f$ is the limit function of some sequence $\left\{f_{n}\right\}$ in $\mathrm{Homeo}_{+}\left(S^{1}\right)$, and $f$ has a jump discontinuity at some point $x$, then we will draw a vertical segment between points $\left(x, f_{-}(x)\right)$ and $\left(x, f_{+}(x)\right)$ as a part of the graph of $f$. 

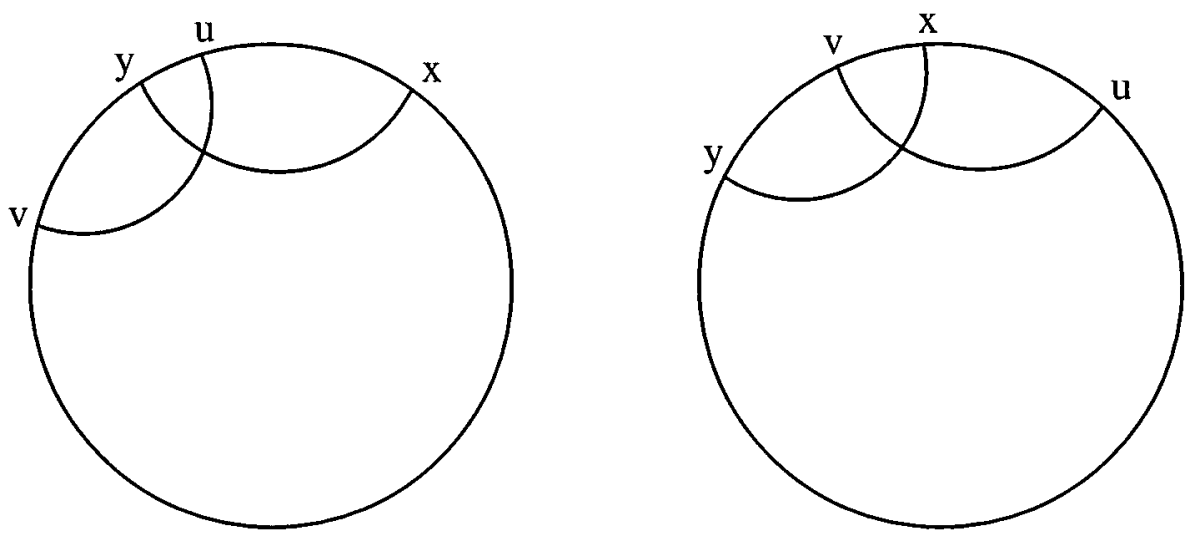

FiguRE 1

Definition. Let $G$ be a subgroup of Homeo+ $_{+}\left(S^{1}\right)$. G is a convergence group ${ }^{2}$ if every sequence $\left\{f_{n}\right\}$ in $G$ has a subsequence $\left\{f_{n_{i}}\right\}$ such that either:

a) $\exists x, y \in S^{1}$ such that

$f_{n_{i}} \rightarrow y$ pointwise on $S^{1}-\{x\}$,

$f_{n_{i}}^{-1} \rightarrow x$ pointwise on $S^{1}-\{y\}$, or

b) $\exists f \in \mathrm{Homeo}_{+}\left(S^{1}\right)$ such that

$f_{n_{i}} \rightarrow f \quad$ pointwise on $S^{1}$,

$f_{n_{i}}^{-1} \rightarrow f^{-1}$ pointwise on $S^{1}$.

In terms of lifts of the $f_{n_{i}}$, condition a) means that the graphs of the $f_{n_{i}}$ approach a sort of step function consisting of jumps and flats (a flat of a function is an interval on which the function is constant) of length 1 . See Figure 2.

Theorem (Tukia, Hinkkanen, Gabai, Casson-Jungreis). G $\hookrightarrow \mathrm{Homeo}_{+}\left(S^{1}\right)$ is conjugate in Homeo $\left(S^{1}\right)$ to a Möbius group if and only if $G$ is a convergence group.

Recall that there are three types of elements of $\mathcal{M}$ which we distinguish by looking at their fixed points on $S^{1}$ : hyperbolic (transformations having two fixed points on $S^{1}$, one attractive, one repulsive; these two points correspond to the endpoints on $S^{1}$ of the hyperbolic axis of the transformation); parabolic (transformations having one fixed point on $S^{1}$ ); elliptic (transformations having no fixed points on $S^{1}$ ).

The number of fixed points is invariant under conjugation, so Möbius-like homeomorphisms can have two, one or no fixed points on $S^{1}$. Accordingly we have the same characterization as for Möbius transformations: a Möbius-like homeomorphism of $S^{1}$ can be either hyperbolic, parabolic or elliptic. However, there is more to the topology of Möbius-like maps than just the number of fixed points, as the following characterization (the proof which can be found in e.g. [T]) shows. Let $f \in \mathrm{Homeo}_{+}\left(S^{1}\right), f \neq i d$.

$f$ has two fixed points on $S^{1}$ : If one fixed point is attractive, usually denoted $P_{f}$, and the other is repulsive, usually denoted $N_{f}$, then $f$ is Möbius-like hyperbolic. In this case we refer to the pair $\left\{N_{f}, P_{f}\right\}$ as the axis of $f$.

\footnotetext{
${ }^{2}$ The original definition of convergence groups is due to Gehring and Martin in [G-M] and it requires all four convergences in conditions a) and b) to be uniform on compact subsets. However it has been shown in $[\mathrm{K}]$ that one can weaken this requirement by asking for pointwise convergence instead. This weakening may not be possible for spheres of higher dimensions.
} 


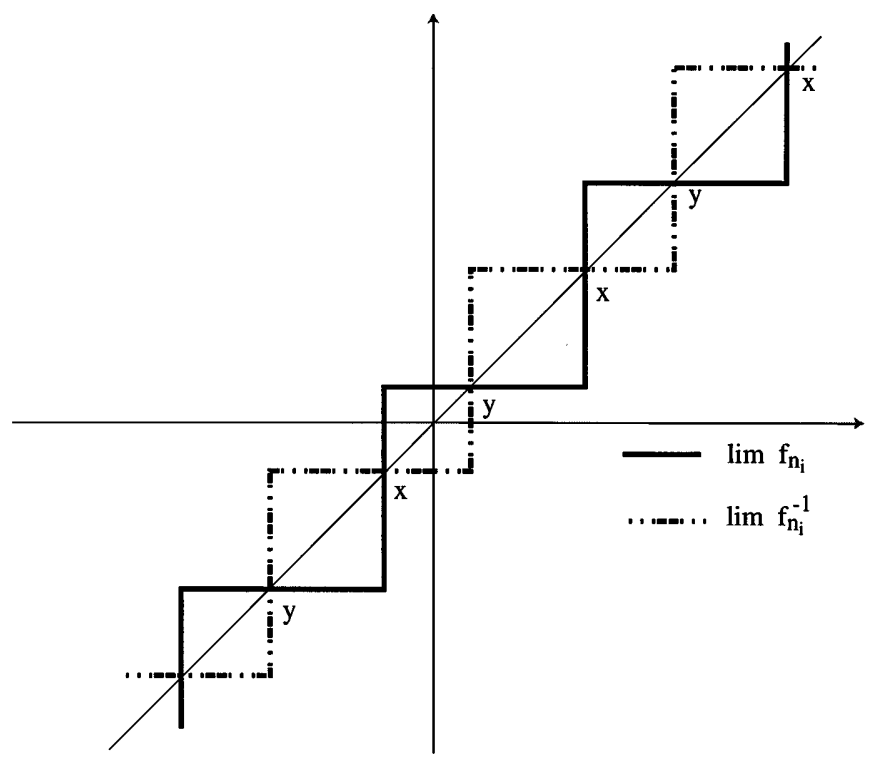

FiguRE 2

$f$ has one fixed point on $S^{1}$ : Then $f$ is necessarily Möbius-like parabolic. $f$ has no fixed points on $S^{1}$ : Then things are more complicated. If $f$ has finite order, i.e., $f^{n}=i d$ for some $n$, then $f$ is Möbius-like elliptic. Now, if $f$ has infinite order, then $f$ needs to satisfy some additional condition in order to be Möbius-like as demonstrated by Denjoy's construction which we recall below. For example, if the $f$-orbit of some point $x \in S^{1}$ is dense in $S^{1}$, then $f$ is Möbius-like elliptic of infinite order.

\section{Denjoy's construction. ${ }^{3}$}

Start with a genuine rotation $f$ of infinite order. Say $f(z)=z e^{2 \pi i \theta}$ with $\theta$ irrational. Choose any $x \in S^{1}$. Then the $f$-orbit of $x, o(x)$, is a countable dense set in $S^{1}$. Now construct a new, bigger circle $\overline{S^{1}}$ by inserting a closed interval at each point of $o(x)$, taking care that the total sum of the lengths of the inserted intervals is finite. See Figure 3.

Now $f$ induces a homeomorphism $\bar{f}: \overline{S^{1}} \rightarrow \overline{S^{1}}$ in the following way: if a point $z \in \overline{S^{1}}$ was untouched by the construction, i.e., no interval was inserted at $z$, set $\bar{f}(z)=f(z)$; to define $\bar{f}$ on the interval inserted at a point $z \in o(x)$, choose any orientation preserving homeomorphism mapping that interval to the interval inserted at the point $f(z) \in o(x)$. Now, $\bar{f}$ has no periodic points, so it cannot be conjugated to a finite order Möbius transformation. On the other hand, it cannot be conjugated to an irrational rotation of $\overline{S^{1}}$ either because there are $\bar{f}$-orbits which are not dense in $\overline{S^{1}}$, and that is a property which is invariant under conjugation.

Definition. Given a Möbius-like group $G$, the limit set of $G$, denoted $L(G)$, is the set of all $x \in S^{1}$ such that $G$ does not act properly discontinuously at $x$ (i.e., for every neighborhood $U$ of $x$ there exist infinitely many elements $g \in G$ such that $g(U) \cap U \neq \emptyset)$. It is easy to see that $L(G)$ is a closed $G$-invariant subset of $S^{1}$.

\footnotetext{
${ }^{3}$ See $[D]$ for more subtleties of the construction, e.g., concerning differentiability.
} 

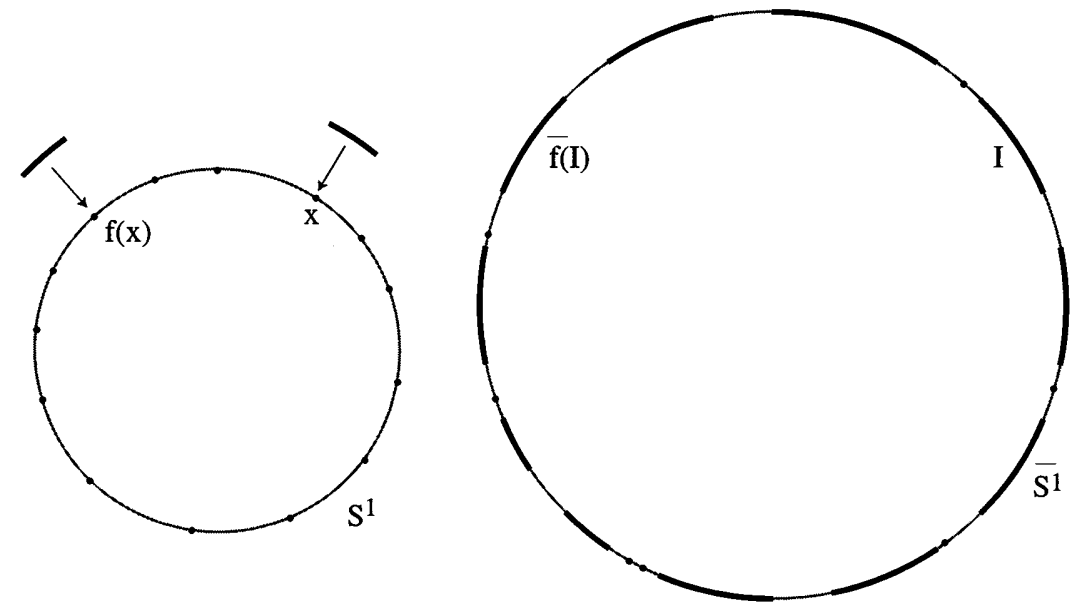

FIGURE 3

Definition ([B]). Let $H$ be a Fuchsian group acting on the open unit disc $\Delta$. A point $x \in L(H)$ is said to be an approximation point of $H$ if there exists a sequence $\left\{h_{n}\right\}$ of distinct functions in $H$ such that for each geodesic half-ray $r$ ending at $x$, there exists a compact subset $K$ of $\Delta$ such that for all $n$

$$
h_{n}(r) \cap K \neq \emptyset .
$$

Lemma. Let $x$ be an approximation point of a Fuchsian group $H$. Then there exists a sequence $\left\{h_{n}\right\}$ in $H$ and two distinct points $a$ and $b$ on $S^{1}$ such that

$$
h_{n} \mid S^{1}-\{x\} \rightarrow b \text { and } h_{n}(x) \rightarrow a .
$$

Proof of lemma. Let $h_{n}$ be the sequence from the above definition. We can assume that, after passing to a subsequence if necessary,

$$
h_{n}(x) \rightarrow a \text { for some } a \in S^{1} .
$$

Since $H$ is a convergence group, we can assume that after passing to a subsequence again, there exist points $b, c \in S^{1}$ such that

$$
h_{n} \mid S^{1}-\{c\} \rightarrow b \text { and } h_{n}{ }^{-1} \mid S^{1}-\{b\} \rightarrow c \text {. }
$$

Clearly, $a \neq b$. For, if $a=b$, then take any $y \in S^{1}-\{c\}, y \neq x$. Then $h_{n}(y) \rightarrow a$, and thus the $h_{n}$ translates of the geodesic line with endpoints $x, y$ limit onto a single point $a$ on $S^{1}$, contrary to the choice of the sequence $\left\{h_{n}\right\}$.

Furthermore, $c=x$. Indeed, if on the contrary $x \in S^{1}-\{c\}$, then $h_{n}(x) \rightarrow b$, which contradicts the fact that $h_{n}(x) \rightarrow a$ and that $a \neq b$.

\section{Construction 1}

In this section we start with a nondiscrete countable elementary Möbius group $H \hookrightarrow \mathrm{Homeo}_{+}\left(S^{1}\right)$; we change its action and add an extra element to obtain a new group $G$ which has properties (a) through (e) as listed in the introduction.

For practical reasons let us be more specific in the choice of $H$ : let us assume that $H$ is a nondiscrete purely elliptic Möbius group whose every element has finite order (e.g., the group generated by rotations $z \mapsto z e^{2 \pi i / n}, n=1,2, \ldots$ ). Choose two points $x, y \in S^{1}$ with disjoint $H$-orbits. Now insert closed intervals into the 


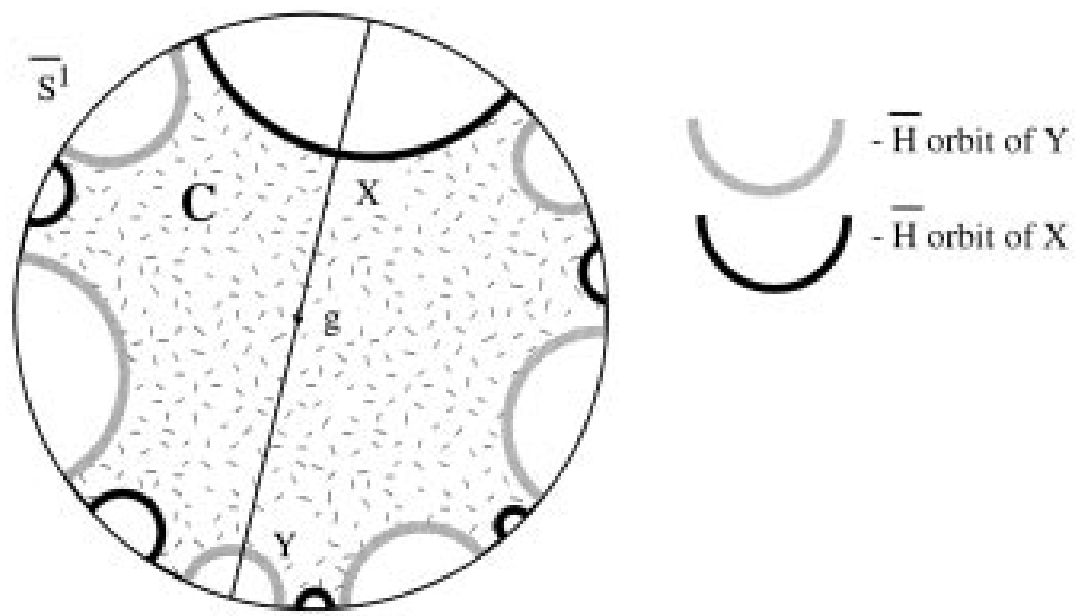

Figure 4

points of the orbits of both $x$ and $y$. As usual, we make sure that the lengths of all the inserted intervals sum to a finite value (this is not a problem since $H$ is countable). This gives us a new, bigger circle which we denote by $\overline{S^{1}}$. We now extend the action of $H$ onto $\overline{S^{1}}$ as follows.

Given $f \in H$, let $\bar{f}$ be a homeomorphism of $\overline{S^{1}}$ induced by $f$ in the following way.

- If $w$ is a point untouched by the construction, i.e., no interval was inserted at $w$, set $\bar{f}(w)=f(w)$.

- Given an interval $[a, b]$ which was inserted at some point $w$ of the original circle, define $\bar{f}$ on $[a, b]$ to be the unique orientation preserving linear map which maps $[a, b]$ onto the interval inserted at $f(w)$.

Set $\bar{H}=\left\{i d_{\overline{S^{1}}}\right\} \cup\left\{\bar{f} \mid f \in G-\left\{i d_{S^{1}}\right\}\right\}$. The use of linear maps for extensions over the inserted intervals guarantees that $\overline{f_{1} \circ f_{2}}=\overline{f_{1}} \circ \overline{f_{2}}$ for any two $f_{1}, f_{2} \in H$. In other words, $\bar{H}$ is a well defined group. Moreover, $\bar{H} \hookrightarrow \mathrm{Homeo}_{+}\left(\overline{S^{1}}\right)$ and all elements of $\bar{H}$ are finite order elliptic Möbius-like homeomorphisms of $\overline{S^{1}}$.

Now draw the geodesic lines $X$ and $Y$ of $\mathbf{H}^{2}$ corresponding to the intervals inserted at $x$ and $y$. See Figure 4 . Let $g$ be a genuine hyperbolic Möbius transformation on $\overline{S^{1}}$ such that $g(X)=Y$ and the axis of $g$ crosses both $X$ and $Y$. Define $G \hookrightarrow \mathrm{Homeo}_{+}\left(\overline{S^{1}}\right)$ to be the group generated by $\bar{H}$ and $g$,

$$
G=\langle\bar{H}, g\rangle .
$$

We now need to see how $G$ acts on $\overline{S^{1}}$, and for that purpose let us denote by $C$ the region bounded by the $\bar{H}$-orbits of $X$ and $Y$ (the shaded region in Figure 4). Then we can say that $\bar{H}$ fixes $C$, i.e., $h(C)=C$ for every $h \in \bar{H}$. Although, strictly speaking, the last statement does not make sense because $\bar{H}$ acts only on $\overline{S^{1}}$ and not on the whole disc, it should be clear what is the corresponding rigorous way of expressing it. The same applies for the considerations which follow. Our main guideline is the idea that we should first completely understand how elements of $G$ act on the region $C$; once that is accomplished, we should be able to understand the entire dynamics of the group $G$. 


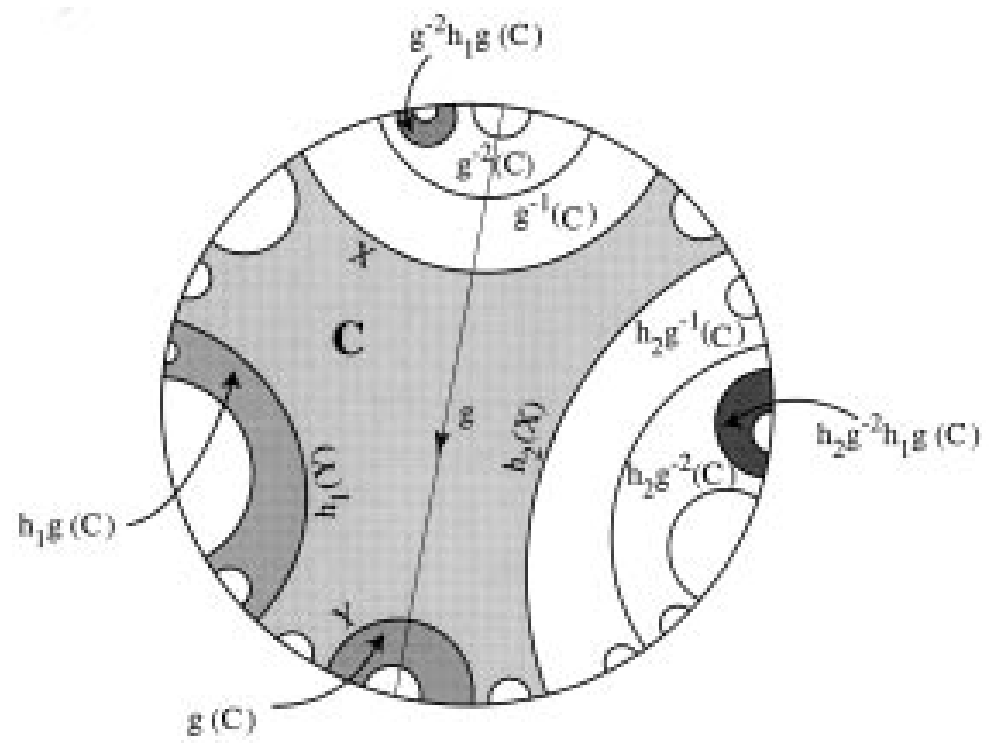

FiguRE 5

Before starting with some observations of a general type, let us consider an example. Let $w_{0}=h_{2} g^{-2} h_{1} g$, where $h_{1}, h_{2}$ are some nontrivial elements of $\bar{H}$. Figure 5 shows how to trace down $w_{0}(C)=h_{2}\left(g^{-2}\left(h_{1}(g(C))\right)\right)$.

Now every element $w$ of $G$ can be represented in the form:

$$
w=h_{k} g^{p_{k}} \ldots h_{2} g^{p_{2}} h_{1} g^{p_{1}},
$$

where $h_{1}, \ldots, h_{k}$ are elements of $\bar{H}$, all nontrivial except possibly $h_{k}$, and $p_{2}, \ldots, p_{k}$ $\neq 0$. Since $X$ and $Y$ come from different orbits of $\bar{H}$, we see that $w(C) \neq C$, unless $w \in \bar{H}$. The above example makes it clear how to trace down $w(C)$ for any given $w$. When presenting the words in $G$ in the form $(*)$, we notice that as the length of presentation grows larger (i.e., $k$ increases), $w(C)$ gets moved "further" 4 from $C$. Thus we have the following two claims

Claim 1. Stabilizer of $C$, the subgroup of $G$ consisting of elements which fix $C$, is exactly equal to $\bar{H}$.

Claim 2. $G$ is a free product of $\bar{H}$ and $\langle g\rangle$, the group generated by $g$.

So the group $G$ defines a sort of tiling of the disc, the tiles being translates of the region $C$ by the elements of $G$. Well, not exactly: we need to check whether the whole disc gets tiled. And indeed that does not have to be the case, but we have the following claim.

Claim 3. We can assume that $G$ tiles the whole disc by the translates of $C$.

Proof of Claim 3. The only type of "anomaly" that stops us from tiling the whole disc is: there exists some interval $[u, v] \subset \overline{S^{1}}$, with corresponding geodesic line $Z$, such that some sequence of translates of $C$ limits onto $Z$ as in Figure 6.

\footnotetext{
${ }^{4}$ We can measure the distance between $C$ and $w(C)$ by counting the least possible number of translates of $C$, by the elements of $G$, one has to pass through on the way from $C$ to $w(C)$. In the above example $\operatorname{dist}\left(C, h_{1} g(C)\right)=1, \operatorname{dist}\left(C, g^{-2}(C)\right)=2, \operatorname{dist}\left(C, w_{0}(C)\right)=3$.
} 


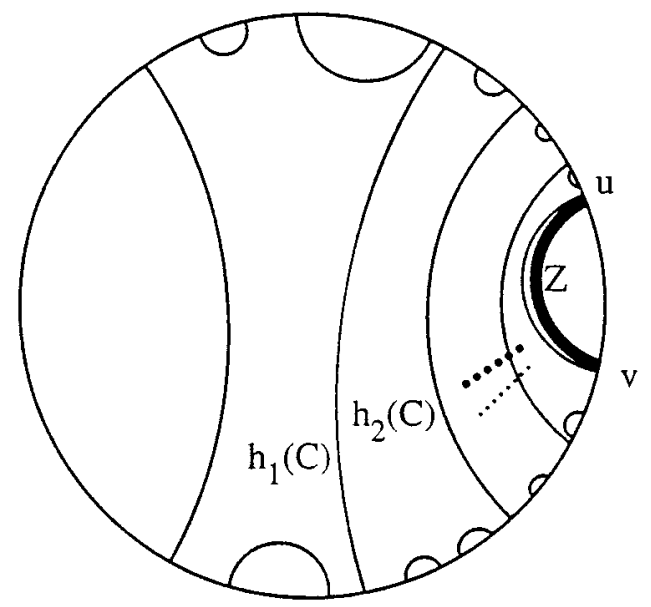

Figure 6

Then no translate of $C$ lives in the region enclosed by $Z$ and $[u, v]$. Clearly, the family $\mathcal{F}$ of all intervals with the same property as $[u, v]$ is a $G$-invariant subset of $\overline{S^{1}}$. It is also clear that the intervals of $\mathcal{F}$ are pairwise disjoint. Therefore we can define yet another circle :

$$
\overline{S_{\star}^{1}}=\overline{S^{1}} /[u, v] \sim \text { point },[u, v] \in \mathcal{F},
$$

with the induced action of $G$ on it. Clearly, this induced action of $G$ on $\overline{S_{\star}^{1}}$ has all the properties of the action of $G$ on $\overline{S^{1}}$ except that every point of $\overline{S_{\star}^{1}}$ is being approached by the translates of $C$. That is exactly what we wanted.

Thus, by replacing $\overline{S^{1}}$ by $\overline{S_{\star}^{1}}$ if necessary, we may assume that given any $z \in \overline{S^{1}}$, there are translates of $C$ which are arbitrarily close to $z$.

Claim 4. Every element of $G$ is Möbius-like.

Proof of Claim 4. Orient the $X$-axis. Let $f$ be an arbitrary nontrivial element of $G$. We want to show that $f$ is Möbius-like, so we distinguish the following cases.

$f$ has one fixed point on $\overline{S^{1}}$ : Then $f$ is Möbius-like as mentioned in the Preliminary Discussions.

$f$ has more than one fixed point on $\overline{S^{1}}$ : The set of all fixed points of $f$ is closed in $\overline{S^{1}}$, so we can find some maximal open interval $(u, v)$ in its complement so that $f$ fixes both $u$ and $v$ and has no fixed points in $(u, v)$. Let $A$ be the geodesic line corresponding to $(u, v)$; we can think of $f$ as fixing $A$. Then some translate of $X$ or $Y$ has to cross $A$. Indeed, if that were not the case, then $A$ would have to be contained in $h(C)$ for some $h \in G$ (because the whole disc is tiled), or possibly $A$ could be in the boundary of $h(C)$. In either case, $f(h(C)) \cap h(C) \neq \emptyset$, so $f \in \operatorname{Stab}(h(C))$. But, by Claim $1, \operatorname{Stab}(h(C))=h \bar{H} h^{-1}$, which is a contradiction because all elements of $h \bar{H} h^{-1}$ are fixed-point free. ${ }^{5}$

\footnotetext{
${ }^{5}$ In case we chose $H$ to be an elementary non-discrete group of a different type, we could say that $f$ is Möbius-like because all elements of $h \bar{H} h^{-1}$ are such.
} 


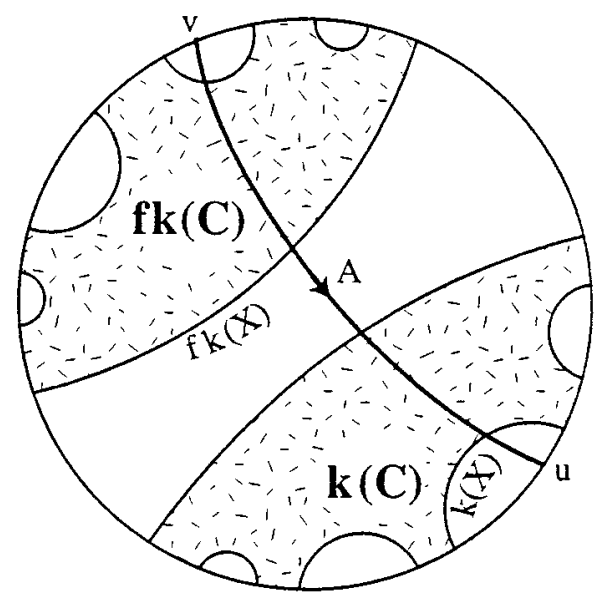

FIGURE 7

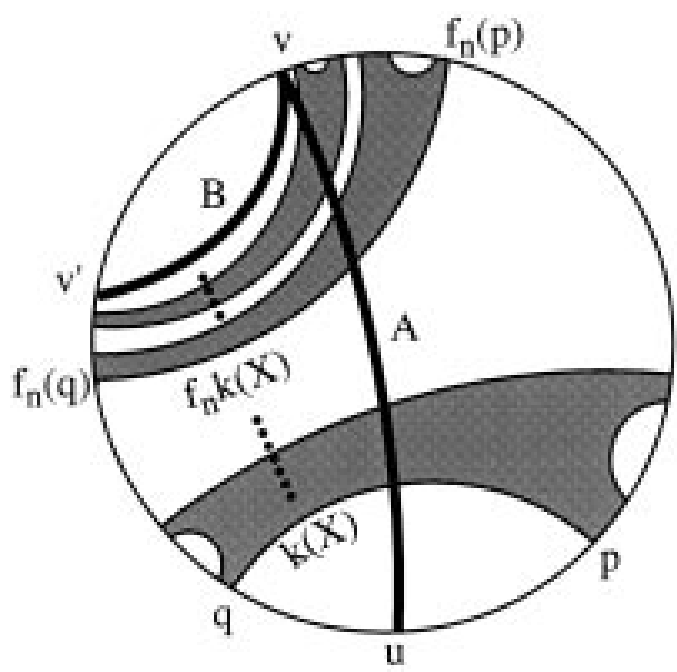

Figure 8

So $A$ crosses some translate of $X$ or $Y$. Without loss of generality we can assume that $A$ crosses $k(X)$ for some $k \in G$. Refer to Figure 7. From the definition of $(u, v)$, it follows that one endpoint of $k(X)$, namely the one lying in $(u, v)$, gets moved under $f$, say towards $v$.

Then, by the tiling property, the whole region $k(C)$ gets moved by $f$ onto the region $f(k(C))$ which is disjoint from $k(C)$. This, we claim, implies that $f$ has no fixed points on $(v, u)$ either. Indeed, to see that let us denote by $p, q$ the endpoints of $k(X)$ which lie in $(u, v),(v, u)$, respectively. Then, it suffices to show that $f^{n}(q) \rightarrow v$ and $f^{-n}(q) \rightarrow u$ when $n \rightarrow \infty$. Suppose the contrary, that say $f^{n}(q) \rightarrow v^{\prime}$ where $v^{\prime} \neq v$. Then $f$ fixes $v^{\prime}$. Refer to Figure 8. Denote by $B$ the geodesic with endpoints $v$ and $v^{\prime}$. 


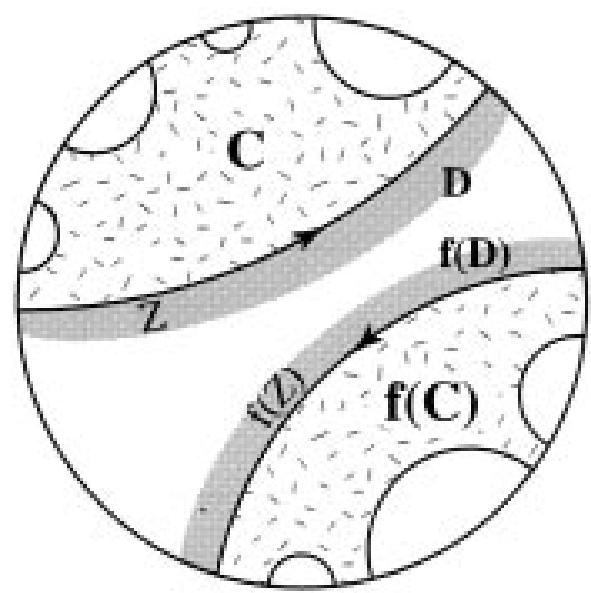

Figure 9

Since $f$ has no fixed points in $(u, v), f^{n}(p) \rightarrow v$. Consequently, the translates of $k(X)$ by positive powers of $f$ are accumulating on $B$. However, since $f$ fixes $B$, the same argument from above used for $A$, we can apply to $B$ to conclude that some translate of $X$ has to cross $B$, and thus it also has to cross $f^{n}(k(X))$ for large enough $n$. Contradiction.

So $u$ and $v$ are the only fixed points of $f$. It is also clear that $f$ has the right dynamics, i.e., one of its fixed points is attractive, while the other is repulsive. Therefore $f$ is Möbius-like hyperbolic.

$f$ has no fixed points on $\overline{S^{1}}$ : Let $h(C)$ be the translate of $C$ which minimizes $\operatorname{dist}(h(C), f(h(C))$ ) (see footnote 2 ). Since conjugation does not change the fact that a map is Möbius-like, we can conjugate $f$ so that $\operatorname{dist}(C, f(C))=d$ is minimal. If $d=0$, then $f \in \operatorname{Stab}(C)=\bar{H}$ and hence $f$ is Möbius-like. Now assume that $d>0$, i.e., $f(C) \neq C$. Assume first that $f(C)$ is not adjacent to $C$ (i.e., $d>1$ ) . Let $Z$ be the boundary component of $C$ which is the closest to $f(C)$. See Figure 9. $f$ has no fixed points so $f(Z)$ must be oriented as in the Figure 9.

But then there exists some translate of $C$ living in the "gap" between $Z$ and $f(Z)$, which decreases $d$, as suggested in Figure 9. Namely, if we denote by $D$ the translate of $C$ which lies along $Z$ only on the opposite side from $C$, then the shortest path between $C$ and $f(C)$ leads through $D$ and $f(D)$, and so $\operatorname{dist}(D, f(D))<d$. Contradiction with the choice of $d$.

Therefore, $f(C)$ must be adjacent to $C$, i.e., they share one boundary component, call it $Z$. See Figure 10.

Then $f^{2}$ fixes both endpoints of $Z$. Consequently $f^{2}=i d$ (if not, then from the above discussion of elements with more than one fixed point it follows that $f^{2}$ would have to be hyperbolic, which is impossible). So $f$ is Möbius-like as stated in the Preliminary Discussions.

So we have proven Claim 4 and hence accomplished our goal: $G$ is a Möbiuslike group, but it is not a convergence group because $\bar{H} \hookrightarrow G$ is not a convergence group. Figure 11 illustrates how the convergence property fails in $G$ i.e., in $\bar{H} \hookrightarrow G$. Namely, we take $h_{n}$ to be any sequence in $H$ which converges to $i d_{S^{1}}$. Then 


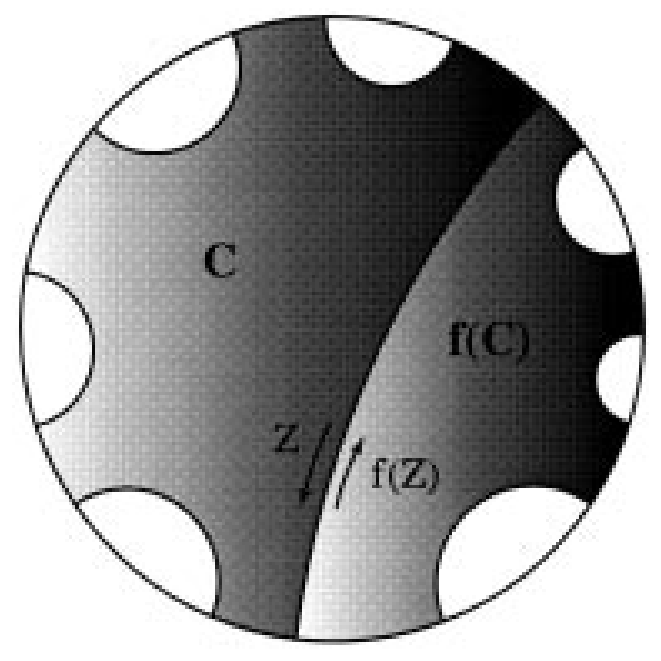

Figure 10
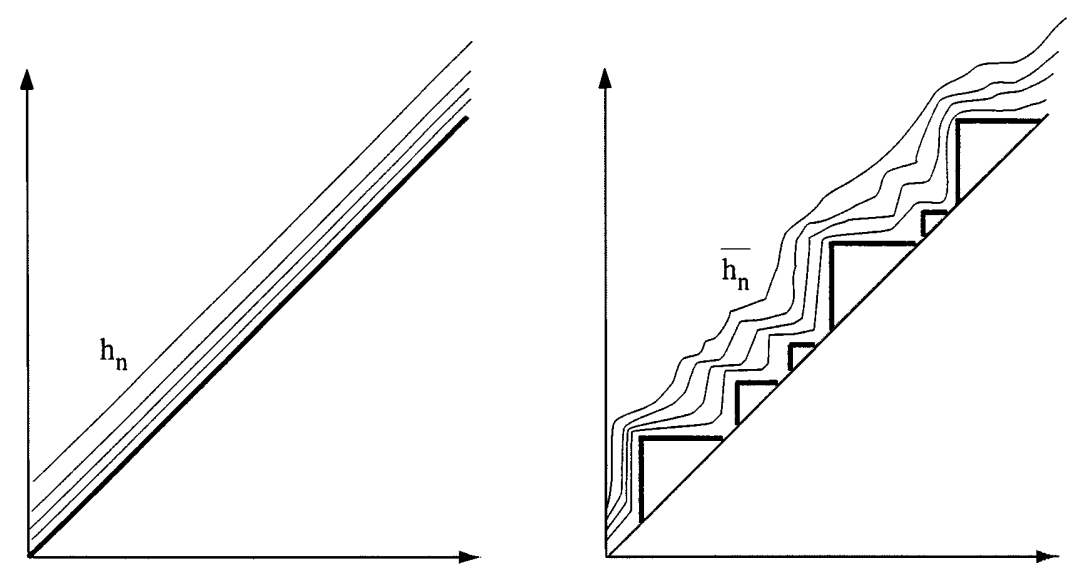

FiguRE 11

the corresponding sequence $\overline{h_{n}}$ in $\bar{H}$, having to preserve the union of the inserted intervals, does not satisfy the convergence property.

Remark. It is clear that the above construction can be varied by changing $H$; any nondiscrete elementary countable group $H$ would do. Care should be taken when "blowing up" orbits of $H$ : one should not insert intervals at points which are fixed points of some element of $H$. In all of these cases the convergence property for $\bar{H}$ and thus $G$ fails in the same a way, i.e., one can find a sequence whose limit function consists of infinitely many triangles determined by the inserted intervals. For example, if interval $[a, b]$ was inserted at some point $w \in S^{1}$, then $h_{n}(w)$ converges to $w$ from above, and thus $\overline{h_{n}}([a, b]) \rightarrow b$. 


\section{Construction 2}

In this section we choose $H$ to be any Fuchsian group of the first kind which has at least one approximation point on the circle which is not a fixed point of any element of $H$.

Denote this approximation point by $x$. Next take $y$ to be any point which is not a fixed point of any element of $H$ and whose $H$ orbit is disjoint from the one of $x$.

Now we can repeat the same procedure used in Construction 1: insert intervals in the orbits of $x$ and $y$ to get $\overline{S^{1}}$ with induced action $\bar{H}$ on it, choose a new element $g$ as before and let $G=\langle\bar{H}, g\rangle$. Note that $H$, being discrete, has no elliptic elements of infinite order and thus $\bar{H}$ is a Möbius-like group. Careful reconsideration of the arguments used in the proofs supporting Construction 1 shows that they are valid in this situation as well. The only difference is at the very end, in showing that $\bar{H}$ and thus $G$ are not convergence groups. To see this, recall the lemma from the end of the Preliminary Discussions which states that we can find some sequence $\left\{h_{n}\right\}$ of distinct elements of $H$ such that

$$
h_{n} \mid S^{1}-\{x\} \rightarrow b, \quad h_{n}(x) \rightarrow a
$$

where $a$ and $b$ are two distinct points on $S^{1}$. We can assume that, after passing to a subsequence, $h_{n}(x)$ converges monotonically (from above or from below) to $a$. Figure 12 shows the possible ways in which the sequence $\left\{h_{n}\right\}$ may approach its limit function.

However, in constructing $\overline{S^{1}}$ we have inserted an interval $J_{x}$ at the point $x$. The corresponding sequence $\left\{\overline{h_{n}}\right\}$ in $\bar{H}$ must preserve the union of all intervals inserted at points from the $H$-orbit of $x$, and thus

$$
h_{n} \mid J_{x} \rightarrow a \text { while } h_{n} \mid \overline{S^{1}}-J_{x} \rightarrow b .
$$

In other words, the limit function of $\left\{h_{n}\right\}$ has two flats and two jumps, as illustrated in Figure 13. This proves that $G$ is not a convergence group.

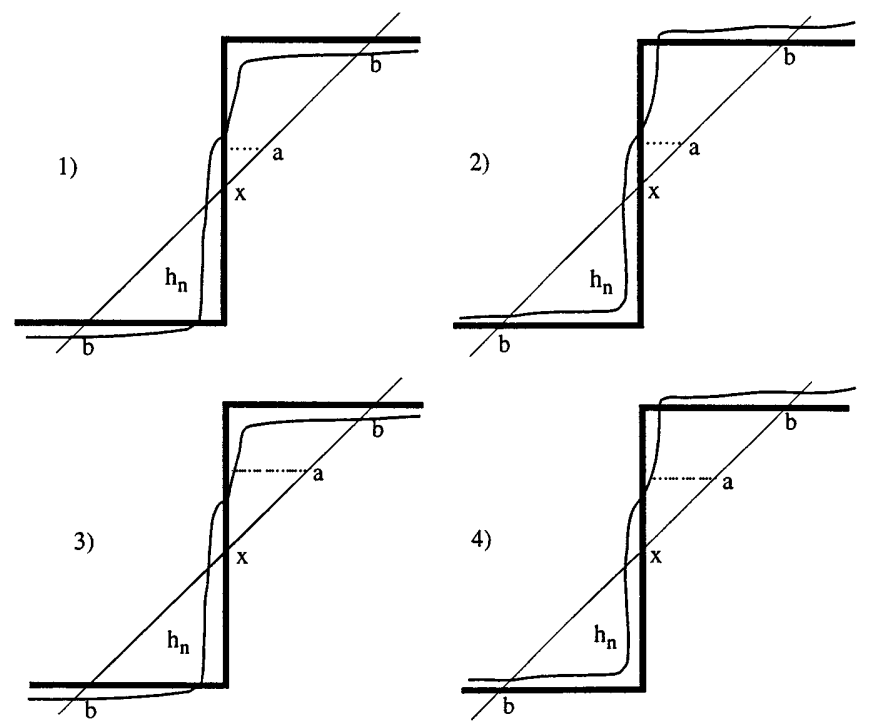

Figure 12 


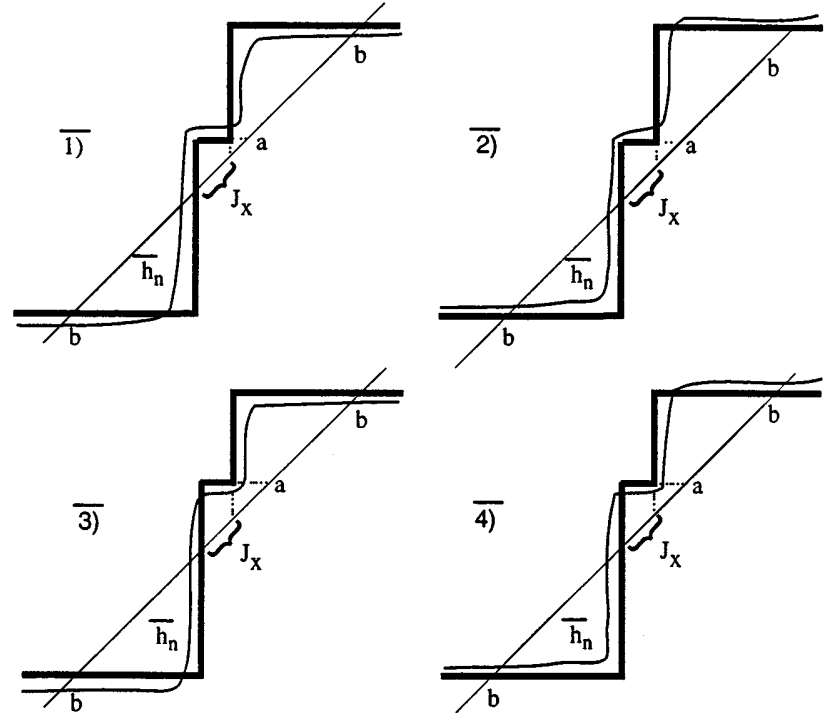

FiguRE 13

\section{REFERENCES}

[B] A. Beardon, The Geometry of Discrete Groups, Springer-Verlag, New York, 1983. MR 85d:22026

[C-J] A. Casson and D. Jungreis, Seifert Fibered Spaces and Convergence Groups, Preprint.

[D] A. Denjoy, Sur les curbes definies par les equations differentielles a la surface du tore, J. Math. Pures Appl. 11 (1932), 333-375.

[G] D. Gabai, Convergence Groups are Fuchsian Groups, Ann. of Math. 136 (1992), 447-510. MR 93m:20065

[G-M] F.W. Gehring and G. Martin, Discrete Quasiconformal Groups, I, Proc. London Math. Soc. 55 (1987) 331-358. MR 88m:30057

[H] A. Hinkkanen, Abelian and Nondiscrete Convergence Groups on the Circle, Trans. A.M.S. 318 (1990), 87-121. MR 91g:30025

[K] N. Kovačević, Möbius-like Groups of Homeomorphisms of the Circle, Trans. A.M.S. 351 (1999).

[T] P. Tukia, Homeomorphic Conjugates of Fuchsian Groups, J. für Reine und Angew. Math. 391 (1988). MR 89m:30047

Department of Mathematics, University of Toronto, 100 St. George Street, Room 4072, Toronto, Ontario M5S 1A1, CAnada

E-mail address: natasak@home.com 